\title{
Design and Analysis of a Novel 16/10 Segmented Rotor SRM for 60V Belt-Driven Starter Generator
}

\author{
Xiaodong Sun ${ }^{1,2 *}$, Zhengwang Xue ${ }^{1,2}$, Shouyi Han $^{3}$, Xing Xu ${ }^{1,2}$, Zebin Yang ${ }^{3}$, and Long Chen ${ }^{1,2}$ \\ ${ }^{1}$ School of Automotive and Traffic Engineering, Jiangsu University, Zhenjiang 212013, China \\ ${ }^{2}$ Automotive Engineering Research Institute, Jiangsu University, Zhenjiang 212013, China \\ ${ }^{3}$ School of Electrical and Information Engineering, Jiangsu University, Zhenjiang 212013, China
}

(Received 12 May 2016, Received in final form 1 September 2016, Accepted 5 September 2016)

\begin{abstract}
This paper proposes a novel 16/10 segmented rotor switched reluctance motor (SSRM) for belt-driven starter generators (BSGs). Different from conventional SRMs, the stator of the proposed SSRM consists of two types of stator poles, i.e., exciting and auxiliary poles, and the rotor is constructed from a series of discrete segments. The topology and operation principle of this proposed SSRM are illustrated firstly, and then the design rules are listed. In addition, the finite element method (FEM) is employed to get the static and dynamic characteristics of the proposed SSRM. Finally, the simulation results are presented to show the validity of the proposed SSRM for BSGs.
\end{abstract}

Keywords : segmented rotor switched reluctance motor, belt-driven starter generator, finite element method, static and dynamic characteristic, simulation result

\section{Introduction}

The belt-driven starter generator (BSG) is gaining more and more attention in hybrid electric vehicles [1-4]. The induction motors (IMs) and permanent-magnet (PM) motors are the prime alternatives to the BSGs. However, as for the IM, it is difficult to acquire the high torque at low speed; regarding the PM motor, its increasing cost of PMs and its demagnetization under high temperature have limited its promotion and degraded its performance [2, 4].

A conventional switched reluctance motor (SRM) is a doubly-salient and single-excited motor where windings are wound in stator poles and there are no windings or PMs in the rotor. The SRM has drawn more and more attention recently due to its remarkable advantages including robustness, low cost and wide applications in serious environment and high speed [5]. Thus, applying the SRM to the BSG under the frequently changing operation circumstances is not only possible but also an academic novelty.

However, the torque ripple, vibration and acoustic noise and low torque to volume are the limitations of the conv-

(C)The Korean Magnetics Society. All rights reserved.

*Corresponding author: Tel: +86-0511-88782845

Fax:+86-0511-88782845, e-mail: xdsun@ujs.edu.cn entional SRM. The torque ripple, vibration and acoustic noise can be reduced by exerting some appropriate control methods [5-10]. In addition to employing the control schemes, modifying the geometry of the SRMs can overcome the above mentioned disadvantages partly as well. In [11], Mecrow et al. propose a novel SRM with full pitch windings to improve the torque density at the expense of the longer end-windings, which is not suitable for short axial length machines. In [12], Z.Y. Xu et al. propose a $6 / 5$ segmental rotor type SRM with single teeth winding. However, the flux of the proposed SRM only flows around one side of the stator and produces a large unbalanced magnetic pull to the rotor, which may increase the acoustic noise and vibration. In this paper, a novel 16/ 10 segmented rotor switched reluctance motor (SSRM) with short, no overlapping end-windings is proposed. The proposed SSRM has the segmented rotor and two types of stator poles, i.e., exciting and auxiliary poles. The static and dynamic characteristics of the proposed SSRM are analyzed by the finite element method (FEM) and the feasibility of the proposed SSRM for BSGs is verified by the simulation results.

\section{Topology and Operation Principle}

A novel 16/10 SSRM is shown in Fig. 1. Different from 


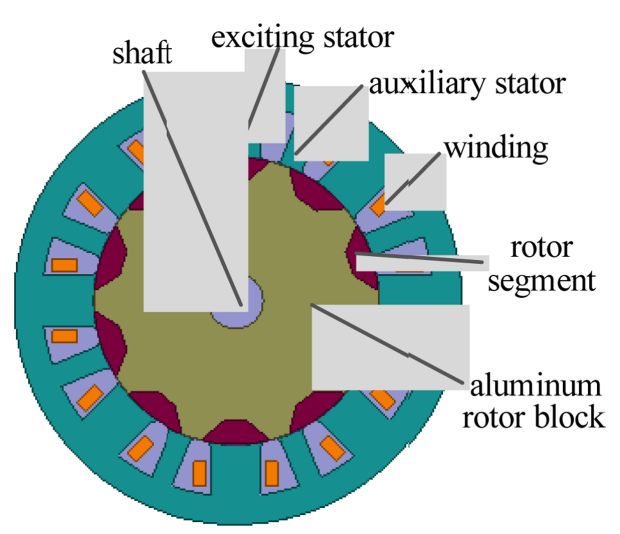

Fig. 1. (Color online) Proposed 16/10 SSRM.

the structure of the conventional SRM, the stator of the proposed SSRM consists of two types of stator poles, i.e., the exciting and auxiliary poles. The exciting poles are wound by the concentrated windings, while the auxiliary poles are not wound by any windings, only providing the flux return path. Moreover, from Fig. 1, it can be seen that the rotor is constructed from a series of discrete segments. Each rotor segment is embedded in an aluminum rotor block without any mechanical saliency, and is magnetically isolated from its neighbors. The new structure of the proposed SSRM can improve its faulttolerant capability effectively.

Figure 2 shows the magnetic flux path of the proposed SSRM. "-" stands for the current flowing into the paper, and "+" stands for the current flowing out of the paper. The winding connection type of the proposed SSRM in this paper is NSNSNSNS. From Fig. 2(a), it can be seen

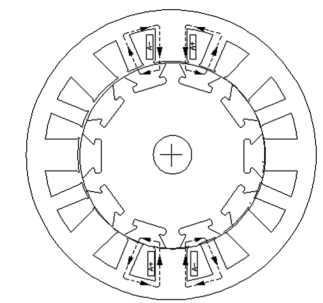

(a)

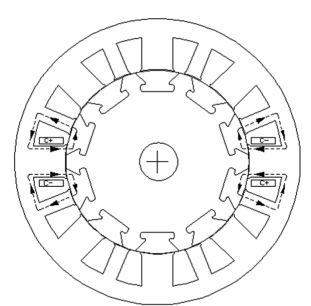

(c)

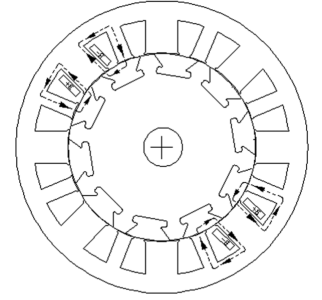

(b)

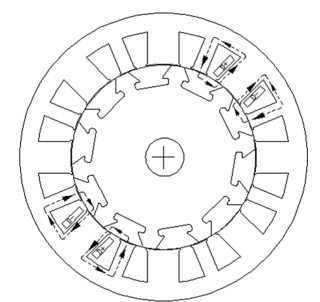

(d)
Fig. 2. Magnetic flux path of the proposed 16/10 SSRM. (a) Phase A excited. (b) Phase B excited.(c) Phase C excited. (d)Phase D excited. that the magnetic flux flows down one stator tooth, through a rotor segment and returns via the adjacent stator tooth. In addition, according to the minimum principle of reluctance, the rotor will rotate in the counterclockwise direction when the phases $\mathrm{D}, \mathrm{A}, \mathrm{B}$ and $\mathrm{C}$ are excited in turn. But the rotor will rotate in the clockwise direction when the phases B, A, D and C are excited in turn.

\section{Design of the Proposed SSRM}

To clarify the structure of the proposed SSRM, the design rules are given as follows:

1. The width of the exciting stator teeth is equal to twice that of the auxiliary stator teeth, as one exciting tooth carries the flux of two auxiliary teeth.

2. The width of the stator slot openings is equal to that of the gap between rotor segments, which ensures the minimum unaligned permeance.

3. Each phase has two coils and they are wound around the opposite exciting stator teeth in parallel. Furthermore, each coil has many turns and each turn will choose many strands, considering selecting the proper wire size and reducing the winding complexity.

Table 1 shows the specifications of the proposed SSRM.

\section{Analysis of the Proposed SSRM}

As is well known, the SRM has the characteristic of high nonlinear flux saturation, and it has switch mode of the main circuit and special control [13]. Therefore, to more accurately analyze the proposed SSRM, the FEM is employed to get the static and dynamic characteristics.

\subsection{Static characteristics}

Table 1. Specifications of the proposed SSRM.

\begin{tabular}{cc}
\hline \hline Parameters & Value \\
\hline Number of phase & 4 \\
Outer radius of stator $(\mathrm{mm})$ & 64 \\
Outer radius of rotor $(\mathrm{mm})$ & 41 \\
Length of stack $(\mathrm{mm})$ & 80 \\
Length of airgap $(\mathrm{mm})$ & 0.25 \\
Yoke thickness of stator $(\mathrm{mm})$ & 8 \\
Stator pole arc $\left({ }^{\circ}\right)$ & $21.375 / 10.69$ \\
Rotor pole arc $\left({ }^{\circ}\right)$ & 26.64 \\
Number of turns & 26 \\
Wire diameter $(\mathrm{mm})$ & 2 \\
Slot factor & 0.82 \\
Resistance per phase $(\mathrm{m} \Omega)$ & 18.5 \\
\hline
\end{tabular}




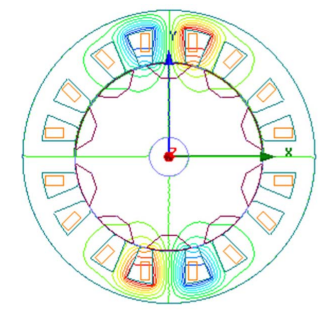

(a)

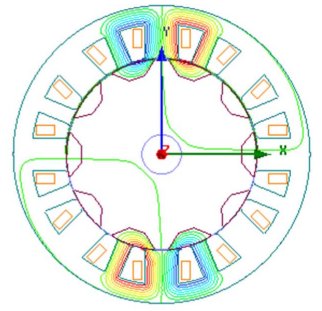

(b)
Fig. 3. (Color online) Magnetic flux distribution of the proposed 16/10 SSRM. (a) Unaligned position. (b) Aligned position.

\subsubsection{Magnetic flux distribution}

Figure 3 shows the magnetic flux distribution of the proposed SSRM when phase A is excited in the aligned and unaligned position. The aligned position is defined as the orientation when one exciting stator pole and the gap between two adjacent rotor segments line up exactly, obtaining the maximum permeance. And the unaligned position is defined as the orientation when one exciting stator pole and one rotor segment line up exactly, attaining the minimum permeance. From Fig. 3, we can see that the magnetic field shows a uniform distribution in the aligned position, and only a small amount of magnetic leakage occurs in the unaligned position. In addition, all conductors in each stator slot only couple with the flux driven by their magneto-motive force (MMF) with a small degree of mutual inductance between one slot and another. That is, the magnetic flux path of each coil is isolated from both that of the other phases and the other coil of the same phase, which may obtain the good faulttolerant capacity.

\subsubsection{Magnetic flux density}

Figure 4 shows the magnetic flux density of the proposed SSRM when phase A is excited in the unaligned and aligned position, respectively. As shown in Fig. 4, when phase $\mathrm{A}$ is excited in aligned position, the average

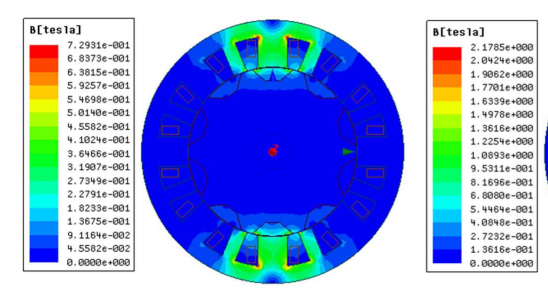

(a)

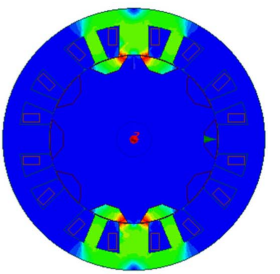

(b)
Fig. 4. (Color online) Magnetic flux density of the proposed 16/10 SSRM. (a) Unaligned position. (b) Aligned position. flux density in the stator yoke is about 1.45T, which satisfies the design requirement. In addition, the flux density in pole tips is more than $1.9 \mathrm{~T}$, but this saturation can be accepted because the saturation in pole tips is inevitable.

\subsubsection{Magnetization curve}

Based on magnetic flux distribution and density, magnetization curve $\psi=f(i, \theta)$ can be obtained from FEM, which is the foundation of the electromagnetic performance analysis. Figure 5 shows the magnetization curve, where the data1 represents zero mechanical degree, data 2 represents two mechanical degrees, data 3 represents four mechanical degrees, ..., and data 9 represents eighteen mechanical degrees. It can be seen from Fig. 5 that the flux linkage of winding $\psi$ shows linear relationship with the phase current $i$ when rotor rotates from 0 mechanical angle degree to 8 mechanical angle degree. But $\psi$ gets to perform nonlinear relationship with the phase current $i$ as mechanical angle $\theta$ increases, which is caused that the flux will be saturated when $\theta$ approaches to the aligned position. In addition, the origin of the horizontal axis starts from $6 \mathrm{~A}$ rather than $0 \mathrm{~A}$, which has almost no influence in the performance of the magnetization curve.

\subsubsection{Inductance profile}

The inductance curves with respect to the rotor position in different phase current levels are shown in Fig. 6. The mechanical degree (mech. deg.) is used in the figure. Since the proposed SSRM has ten rotor segments, a rotation of $18^{\circ}$ from unaligned position to aligned position consists of half mechanical cycle. From Fig. 6, it reveals that in one phase current level, phase inductance increases gradually as the rotor approaches to the aligned position $\left(18^{\circ}\right)$, but tends to be a flat due to saturation in the aligned position. Moreover, in different current levels, the

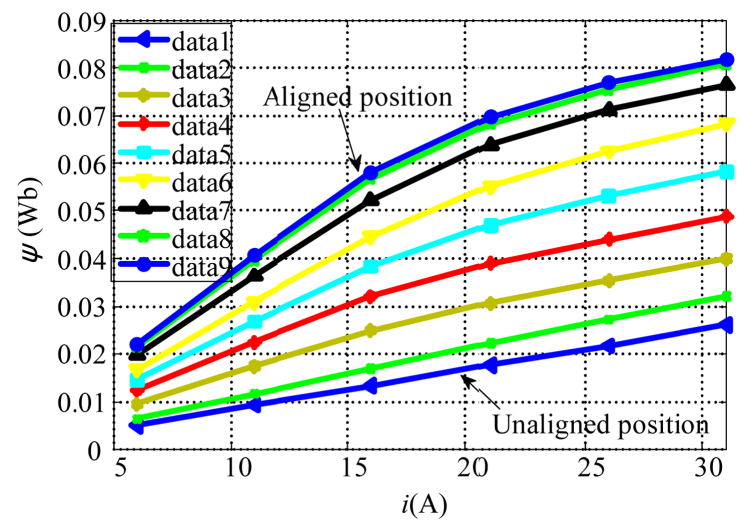

Fig. 5. (Color online) Magnetization curve of the proposed 16/10 SSRM. 


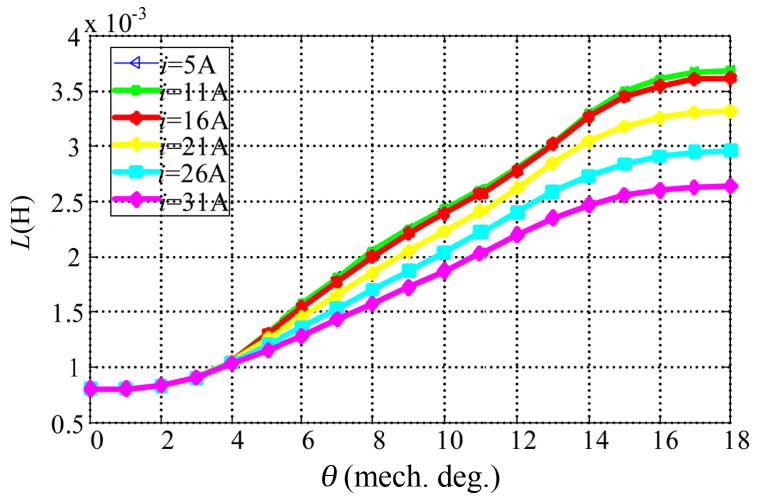

Fig. 6. (Color online) Inductance profile of the proposed 16/ 10 SSRM.

phase inductance is inversely proportional to current. That is, the higher current value is given, the lower inductance value is obtained.

\subsubsection{Torque profile}

The torque of a SRM is produced by the attraction from the exciting stator pole to the rotor pole in order to maximize the permeance [14]. Thus, the torque, which is related to phase current and the rotor position, can be expressed as

$$
T=\frac{1}{2} i^{2} \frac{\partial L(i, \theta)}{\partial \theta}
$$

where $T$ is the torque, $i$ is the phase current, $L$ is the phase inductance, and $\theta$ is the rotor position angle.

The torque curves with respect to the rotor position in different phase current levels are shown in Fig. 7. When the currents are small, the proposed SSRM is operating in a linear model, and on the condition that the values of the currents are high, the torque with respect to the rotor

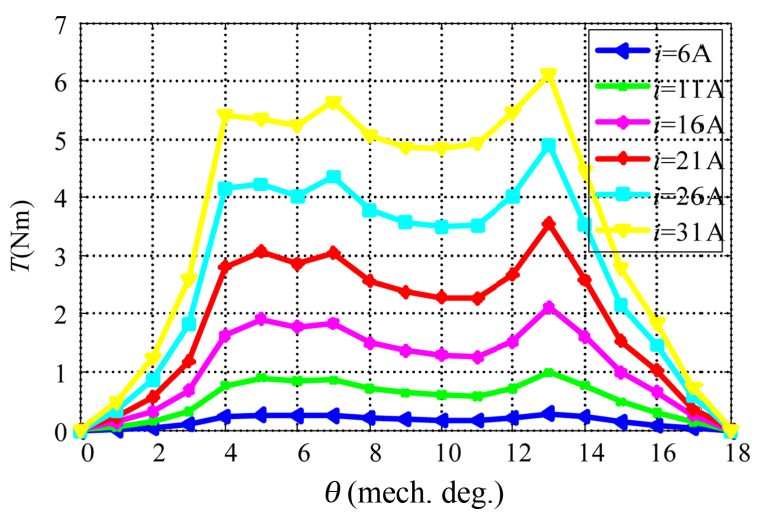

Fig. 7. (Color online) Torque profile of the proposed 16/10 SSRM.

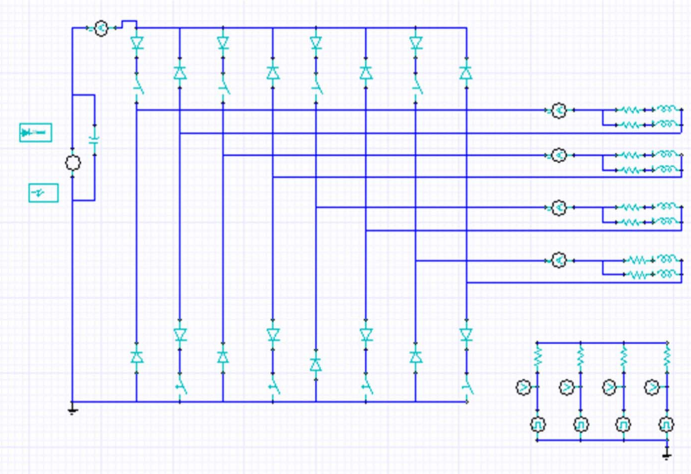

Fig. 8. (Color online) Four-phase asymmetric half-bridge converter.

position is almost sinusoidal in shape. Furthermore, the higher current value is given, the bigger torque is attained, which corresponds to the equation (1).

\subsection{Dynamic characteristics}

\subsubsection{Asymmetric converter}

The proposed SSRM is coupled to a four-phase asymmetric half-bridge converter from Ansoft Maxwell. Figure 8 shows the topology of this converter circuit. It includes half-bridge converter, the motor winding circuit and voltage control circuit. The turn on and turn off angles are respectively $-4^{\circ}$ and $12^{\circ}$ at the rated speed of $6000 \mathrm{r} / \mathrm{min}$ under angle position control. When the motor runs at high speed, angle position control is used to change the switching time by adjusting the turn-on angle and turn-off angle. And the chopped current control introduced in the next part is used in the application where the motor starts up. In this way, the electromotive force is low, and the phase current will rise quickly, which may destroy the power switch. Thus, the angle position control is used to limit the peak value of the current.

\subsubsection{Transient current and torque}

Figure 9 shows the simulated transient current and torque waveforms with the proposed SSRM operating at the rated speed of $6000 \mathrm{r} / \mathrm{min}$. The SSRM operates under angle position control, with each phase conducting for about half of a mechanical cycle. As can be seen in Fig. 9(a), the current waveform is regular, although the value of the current is relatively low at the beginning. This can be explained that the current waveform fluctuates and the value is low when the current begins to build, and effective current value is about 26A. Besides, the average torque is up to about $3.1 \mathrm{Nm}$ shown in Fig. 9(b), which satisfies the requirement of the rated torque. 


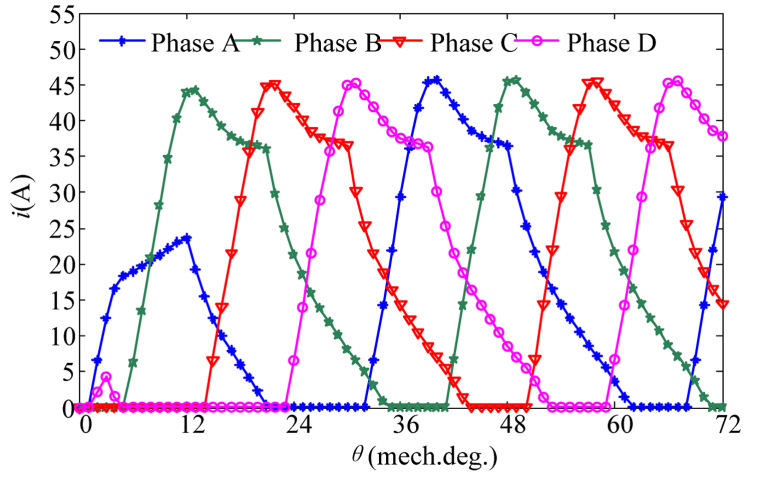

(a)

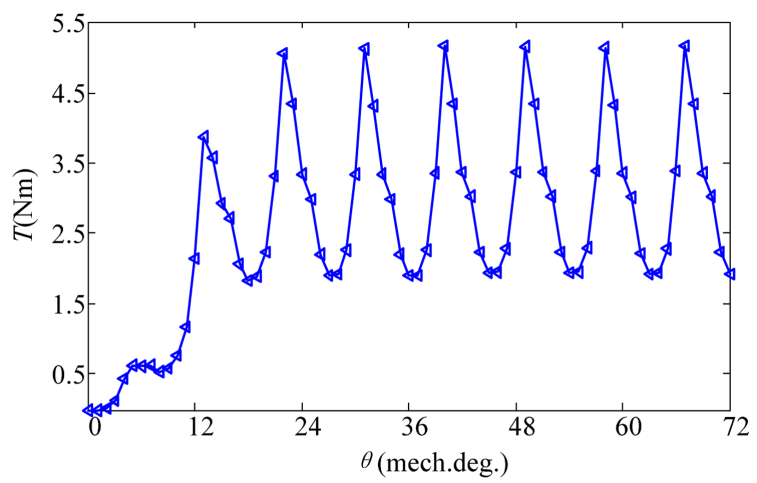

(b)

Fig. 9. (Color online) Transient current and torque waveforms at rated speed. (a) Transient current waveform. (b) Transient torque waveform.

\section{Feasibility of the Proposed SSRM Being Applied to BSG}

The good electromagnetic performance of the proposed SSRM has been investigated and validated above, thus it is natural and promising for the proposed SSRM to examine its feasibility in the BSG.

The studied BSG in this paper is a 60 -VDC system that performs two main functions. The first function (0-6000 $\mathrm{r} / \mathrm{min}$ ) is to start the engine with at least $25 \mathrm{Nm}$ torque, and the second function $(6000-16000 \mathrm{r} / \mathrm{min})$ is to generate $1.8 \mathrm{KW}$ and $60 \mathrm{~V}$ power and then is reduced to $12 \mathrm{~V}$ system to provide power for battery and other application devices in the hybrid electric vehicles through DC/DC inverter.

Due to the proposed SSRM possessing the symmetric structure, the electromagnetic performance in starting condition is theoretically consistent with that in generating condition. Therefore, the simulated transient torque at the rated speed of $6000 \mathrm{r} / \mathrm{min}$ above satisfies the power requirement in generating condition. In addition, the simulated induced voltage from Ansoft is shown in Fig. 10. From Fig. 10, we can see that the simulated value is

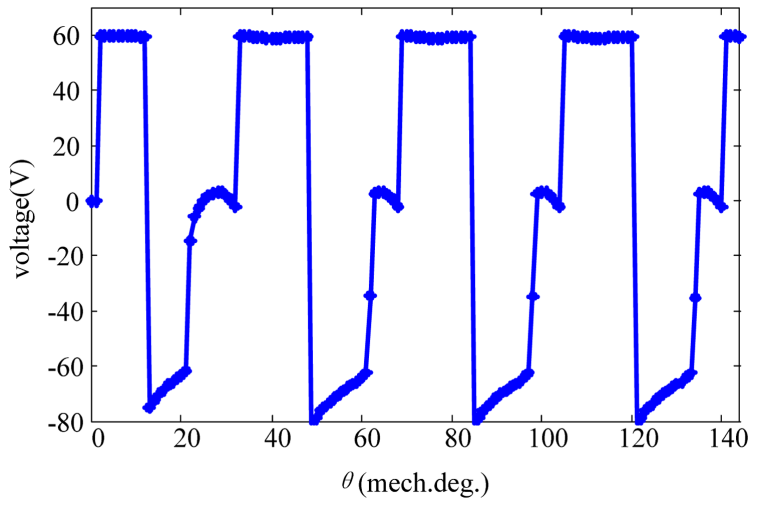

Fig. 10. (Color online) Induced voltage from Ansoft.

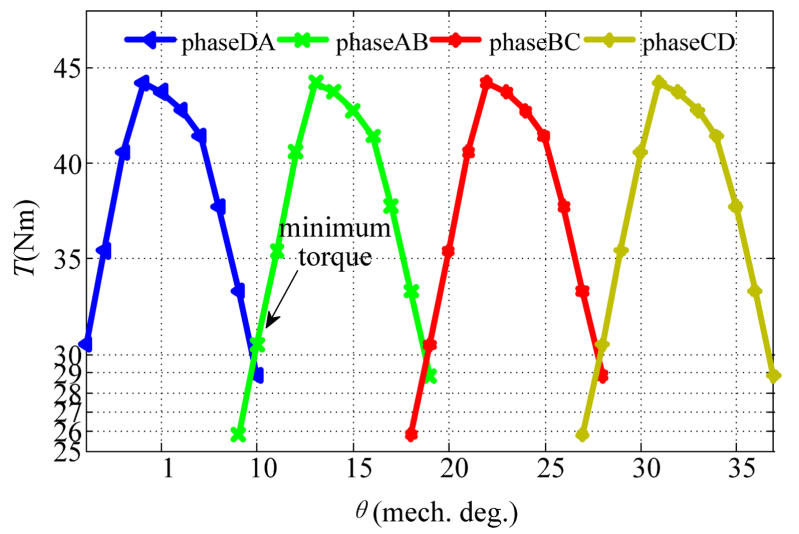

Fig. 11. (Color online) Starting torque of the proposed SSRM.

about $60 \mathrm{~V}$, which also meets the demand of the voltage.

When starting the engine, the BSG is operated under chopped current control [14] and meanwhile two phase are excited simultaneously. The chopped current is about 200A considering starting the engine instantaneously. And then the static torque in starting condition is obtained from Ansoft, which is shown in Fig. 11. From Fig. 11, it is obvious that the minimum starting torque $T_{s t(\min )}$ is up to about $29 \mathrm{Nm}$, which meets the starting torque requirement.

\section{Conclusions}

In this paper, a novel $16 / 10 \mathrm{SSRM}$ is proposed for the BSG of hybrid electric vehicles. The design of this proposed SSRM is illustrated and the static and dynamic characteristics are analyzed. Based on its good electromagnetic performance, the validity of the proposed SSRM for BSGs is verified by the simulation results. It reveals that whether in starting condition or in generating condition, the requirements of torque and voltage are satisfied. Meanwhile, the prototype of the SSRM is being manu- 
factured. This paper also lays the theoretical foundation on the optimal design of the SSRMs and further experimental studies.

\section{Acknowledgments}

This work was supported in part by the National Natural Science Foundation of China under Projects 51305170, U1564201, 51475214, 51405203, 51575240, and 51475213, by the National Science Foundation of Jiangsu Province under Projects BK20130515 and BK20141301, by the China Postdoctoral Science Foundation under Project 2015T80508, by the Six Categories Talent Peak of Jiangsu Province under Projects 2015-XNYQC-003 and 2014-ZBZZ-017, by the Priority Academic Program Development of Jiangsu Higher Education Institutions (PAPD), and by the Synergistic Innovation Center of Modern Agricultural Equipment and Technology in Jiangsu Province.

\section{References}

[1] B. Fahimi, A. Emadi, and B. Raymond, IEEE Trans. Energy Conversion. 19, 1 (2004).

[2] G. H. Lee, H. H. Lee, and Q. Wang, J. Magn. 18, 4
(2013).

[3] W. Ding and D. Liang, IEEE Trans. Energy Conversion 25, 4 (2010).

[4] S. Chen, B. Lequesne, R. R. Henry, Y. Xue, and J. J.Ronning, IEEE Trans. Ind. Appl. 38, 6 (2002).

[5] G. Li, J. Ojeda, S. Hlioui, E. Hoang, M. Lecrivain, and M. Gabsi, IEEE Trans. Magn. 48, 6 (2012)

[6] D. H. Lee, J. N. Liang, Z. G. Lee, and J. W. Ahn, IEEE Trans. Ind. Electron. 56, 8 (2009).

[7] X. Zhang, X. Wang, Y. Yang, and B. Wei, Proceedings of the CSEE. 35, 6 (2015).

[8] M. Takiguchi, H. Sugimoto, N. Kurihara, and A. Chiba, IEEE Trans. Energy Conversion. 30, 3 (2015).

[9] S. R. Mousavi-Aghdam, M. R. Feyzi, N. Bianchi, and M. Morandin, IEEE Trans. Ind. Electron. 63, 3 (2016).

[10] Y. H. Hu, C. Gan, W. P. Cao, Y. T. Fang, S. J. Finney, J. and $\mathrm{H}$. Wu, IEEE Trans. Ind. Appl. 52, 4 (2016).

[11] B. C. Mecrow, J. W. Finch, E. A. EI-Kharashi, and A. G. Jack, IEEE Proc. Electr. Power. 149, 4 (2002).

[12] Z. Y. Xu, D. H. Lee, and J. W. Ahn, Industry Applications Society Annual Meeting (2015).

[13] W. Ding and D. Liang, IEEE Trans. Magn. 44, 7 (2008).

[14] W. Ding, D. Liang, and Z. Cheng, IEEE International Conference on Mechatronics and Automation (2007). 